\title{
Energy-varying beam extraction assisted by large momentum deviation and charge exchange
}

\author{
Ken Takayama, Tadamichi Kawakubo, and Toshikazu Adachi \\ High Energy Accelerator Research Organization (KEK), Accelerator Laboratory, \\ Tsukuba, Ibaraki 305-0801, Japan \\ Tanuja Dixit@ and Aqsa Shaikh@ \\ Society for Applied Microwave Electronics Engineering and Research (SAMEER), Mumbai 400076, India
}

(Received 17 February 2020; accepted 21 December 2020; published 11 January 2021)

\begin{abstract}
Two energy-varying beam extraction schemes for energy sweeping compact rapid cycling therapy are discussed. One is one-turn (fast) extraction of a $\mathrm{C}^{6+}$ bunch from a fast-cycling hadron driver accelerator assisted by an off-momentum bump orbit, in which a combination of a kicker and a septum magnet are fired at a desired timing (or energy). The other is energy sweeping extraction, in which a $\mathrm{C}^{5+}$ bunch delivered from the laser ablation ion source is injected and accelerated in the barrier bucket up to the final energy. A fraction of the trapped $\mathrm{C}^{5+}$ ions is continuously released from the bucket on the way to the final energy to form a coasting beam over an expected time (or energy) region, and the beam drifts inward in the large flat momentum dispersion region. The drifting $\mathrm{C}^{5+}$ beam hits the stripper carbon foil located at the front of the bending magnet and near the inner surface of the vacuum chamber in the large flat dispersion region and is converted to $\mathrm{C}^{6+}$ beam. The combination of the enhanced bending radius and septum magnet allows easy $\mathrm{C}^{6+}$ beam extraction by the single septum magnet downstream.
\end{abstract}

DOI: 10.1103/PhysRevAccelBeams.24.011601

\section{INTRODUCTION}

Energy sweeping compact rapid cycling hadron therapy (ESCORT) [1] based on a fast-cycling induction synchrotron [2] has been proposed for the next generation of hadron therapy, and KEK and SAMEER are currently collaborating on the engineering design work [3]. ESCORT has two notable features: A hadron beam bunch is extracted in one turn at an arbitrary timing and with a desired energy, and a continuous beam is extracted for a desired duration with an expected energy variation in time. It is crucial that the extraction timing and energy in the one-turn extraction and the extraction time duration and energy range in the energy sweep extraction can be changed cycle by cycle, although the driver accelerator is always operated in the same repetition pattern of $10 \mathrm{~Hz}$. Generally, a combination of a kicker magnet and a septum magnet is used for the oneturn extraction of a $\mathrm{C}^{6+}$ beam. Beam extraction at an arbitrary timing should be possible if the excitation currents for the kicker and septum magnet and their firing are controlled. The original circuit architecture of their power

Published by the American Physical Society under the terms of the Creative Commons Attribution 4.0 International license. Further distribution of this work must maintain attribution to the author(s) and the published article's title, journal citation, and DOI. supplies has been developed [4], and the details are discussed below.

The ESCORT driver ring lattice $[1,3]$ is specific. The region for extraction is characterized by a large dispersion function $D(s)$ of 3-6 m. For one-turn extraction, a hadron bunch captured in the barrier bucket is slightly overaccelerated by appropriate handling of the induction acceleration cells. Combining the large dispersion function and offmomentum acceleration of $\Delta p / p=+0.2 \%$ just before the extraction timing generates a bump orbit, $D(s) \Delta p / p$, that improves kicker and septum performance. Basic features in the extraction process using a combination of kicker and septum magnet are similar to the usual fast extraction from a conventional fast-cycling rf synchrotron, except for the circuit architecture of the kicker and septum magnet power supplies. This novel circuit architecture is crucial for energy-varying extraction that must always follow the excitation profile of the main magnet fields of the ring. This allows the extracted beam center to always be placed at the same center through the beam line downstream.

In the original energy sweeping extraction design, a combination of a voltage-varying electrostatic septum and Lambertson magnet was assumed as the extraction system. However, to our knowledge, voltage varying in the range of $50-120 \mathrm{kV}$ at $10 \mathrm{~Hz}$ has not been demonstrated yet, and further research and development is required. As an alternative scheme, energy sweeping extraction using 


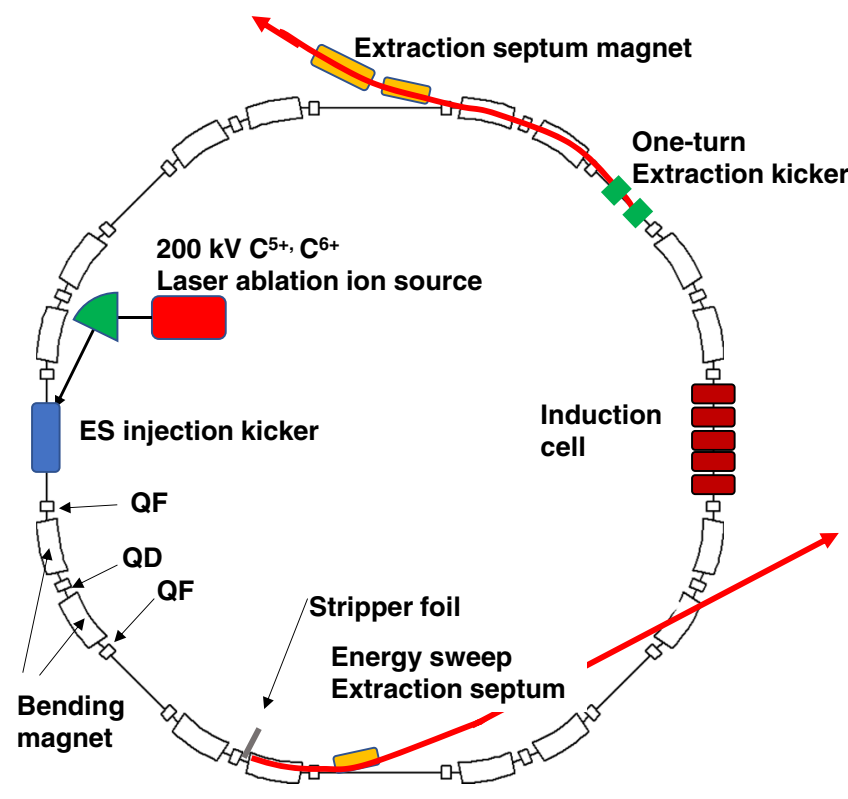

FIG. 1. Lattice of the ESCORT ring.

charge conversion from $\mathrm{C}^{5+}$ to $\mathrm{C}^{6+}$ is proposed. For this purpose, a carbon stripping foil is introduced. In this scheme, a $\mathrm{C}^{5+}$ bunch delivered from the laser ablation source is captured in the barrier bucket, and a fraction of the accelerated $\mathrm{C}^{5+}$ bunch is released by specific barrier bucket handling, as discussed in Ref. [1]. The $\mathrm{C}^{5+}$ beam forms an off-momentum coasting beam that gradually drifts inward in the large momentum dispersion region. Eventually, the beam hits the stripping foil placed near the inner surface of the vacuum chamber between the defocusing quadrupole magnet and the bending magnet (Fig. 1). The orbit of the converted $\mathrm{C}^{6+}$ beam is dramatically changed as it passes through the downstream bending magnet. Consequently, the orbit separation for extraction is sufficient for the septum magnet to work. The feasibility of energy sweep extraction associated with charge conversion is examined here.

It is important that energy sweeping extraction is performed in any time region or a different energy range within the same acceleration cycle. The extracted $\mathrm{C}^{6+}$ beam can sweep a tumor from its front edge to its other edge at $10 \mathrm{~Hz}$ without causing undesired activation around the irradiation device. We stress that this quick irradiation is much faster than natural movement of an organ associated with respiration over 4 or $5 \mathrm{~s}$. Integration with a fast feedback deflector in the transverse direction [4], real-time monitoring of the tumor profile [5], and a dose profile detector [6] will allow continuous irradiation without dead time in respiration-synchronized irradiation, which is the most common technique in present hadron therapies. In addition, beam delivery in this style should become inevitable to ensure uniform irradiation in a possible next generation of hadron therapy without a gantry, where the
TABLE I. Machine and beam parameters.

\begin{tabular}{lcc}
\hline \hline Injection energy & $E_{\text {in }}$ & $<0.1 \mathrm{MeV} / \mathrm{au}$ \\
Extraction energy & $E_{\text {out }}$ & $200-430 \mathrm{MeV} / \mathrm{au}$ \\
Circumference & $\mathrm{C}_{0}$ & $70.64 \mathrm{~m}$ \\
Maximum bending field & $B_{\max }$ & $1.2 / 1.5(\mathrm{~T})$ \\
Bending radius & $\rho$ & $4.429 \mathrm{~m}$ \\
Betatron tune & $Q_{x} / Q_{y}$ & $1.88 / 1.697$ \\
Momentum compaction factor & $\alpha_{p}\left(1 / \gamma_{T}^{2}\right)$ & 0.0396 \\
Transition energy & $\gamma_{T}$ & 5.02 \\
Natural chromaticity & $\xi_{x} / \xi_{y}$ & $-1.65 /-0.047$ \\
Particle number (shot/s) & $N$ & $4 \times 10^{8} / 4 \times 10^{9}$ \\
Beam emittance for $1 \sigma$ & $\varepsilon_{x} / \varepsilon_{y}$ & $40 \mu \mathrm{m} \mathrm{rad}$ \\
Revolution frequency & $f$ & $<3.12 \mathrm{MHz}$ \\
\hline \hline
\end{tabular}

tumor position and profile are changed due to gravitation when a patient in a couch or bed is rotated in the 3D directions.

For the convenience of readers, the basic machine and beam parameters of the ESCORT ring [3] are listed in Table I.

\section{OFF-MOMENTUM BUMP ORBIT FOR ENERGY-VARYING ONE-TURN EXTRACTION}

The lattice and lattice functions of the ESCORT driver accelerator are shown in Figs. 1 and 2, which has been modified from the original design given in Ref. [1]. The main difference is its circumference, which allows the sufficiently long straight sections to accommodate the injection or extraction and acceleration devices. Increasing of the circumference demands a larger acceleration voltage per turn. This is easily solved by increasing the number of induction cells with an output voltage of $2-3 \mathrm{kV} /$ cell. Some regions of the $2.5-\mathrm{m}$-long straight section and the 4-m-long straight section are occupied by the kicker magnet and septum magnet system, respectively.

The off-momentum acceleration of the barrier-trapped $\mathrm{C}^{6+}$ bunch is turned on slightly earlier than the desired



FIG. 2. Lattice functions and extraction device regions for kicker, carbon foil, and septum magnet (note that two extraction schemes are depicted in this figure). 


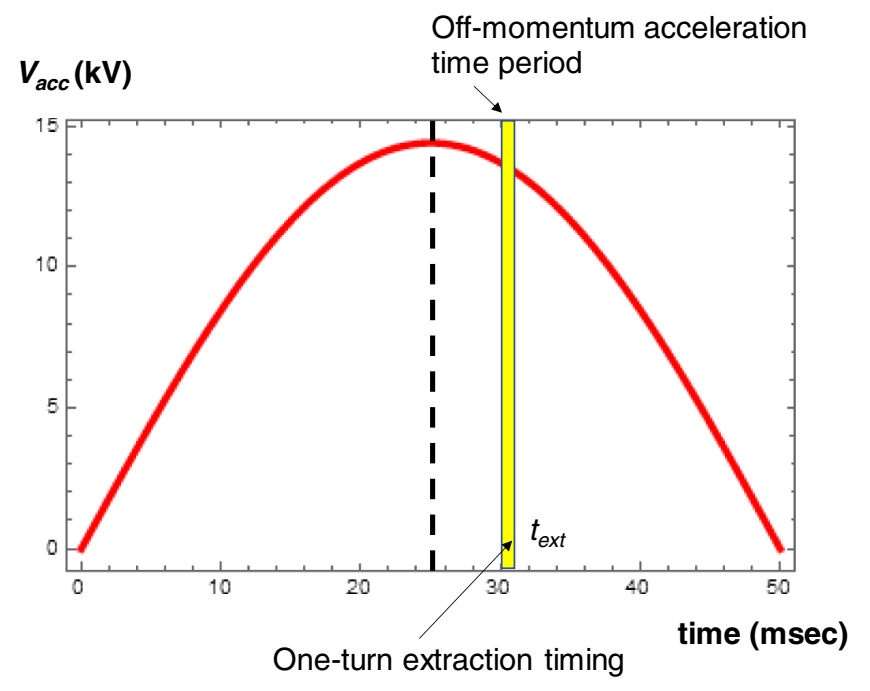

FIG. 3. Required acceleration voltage profile and off-momentum deceleration time period (yellow).

extraction timing $t_{\mathrm{ext}}$ and continues until the bunch center achieves $\Delta p / p=0.20 \%$ at $t=t_{\mathrm{ext}}$. Eventually, a bump orbit of $D(s) \Delta p / p \sim 0.7-1.4 \mathrm{~cm}$ is created in the short and long straight sections for extraction. This size of orbit bump can assist the one-turn extraction kickers greatly.

The off-momentum acceleration to guarantee the momentum deviation of the bunch center of $\Delta p / p=$ $0.2 \%$ is realized by the following procedures.

(i) An idling induction or additional cell that is not involved beyond the full operation stage of all induction cells, shown as the center of the required acceleration voltage profile, is turned on, depending on $t_{\mathrm{ext}}$ (Fig. 3). The induced voltage on the cell gives extra energy to the $\mathrm{C}^{6+}$ bunch during off-momentum acceleration. In the example shown in Fig. 3, a time period for off-momentum acceleration is typically $0.25 \mathrm{~ms}$ ( $\sim 500$ turns), assuming an extra acceleration voltage of $3 \mathrm{kV}$ per turn, where the momentum deviation linearly increases in time as seen Fig. 4. (ii) The induction cell is triggered every turn until the position monitor on the long straight section (iii) detects a signal of $D(s) \Delta p / p \sim 1.4 \mathrm{~cm}$. (iv) The two kickers that are placed on the upper 2.5-m-long straight section are fired and kick the $\mathrm{C}^{6+}$ bunch outward slightly.

The extraction orbit and parameters of the extraction devices have been optimized. A typical extraction orbit is shown in Fig. 5. Details of the kicker magnets and septum

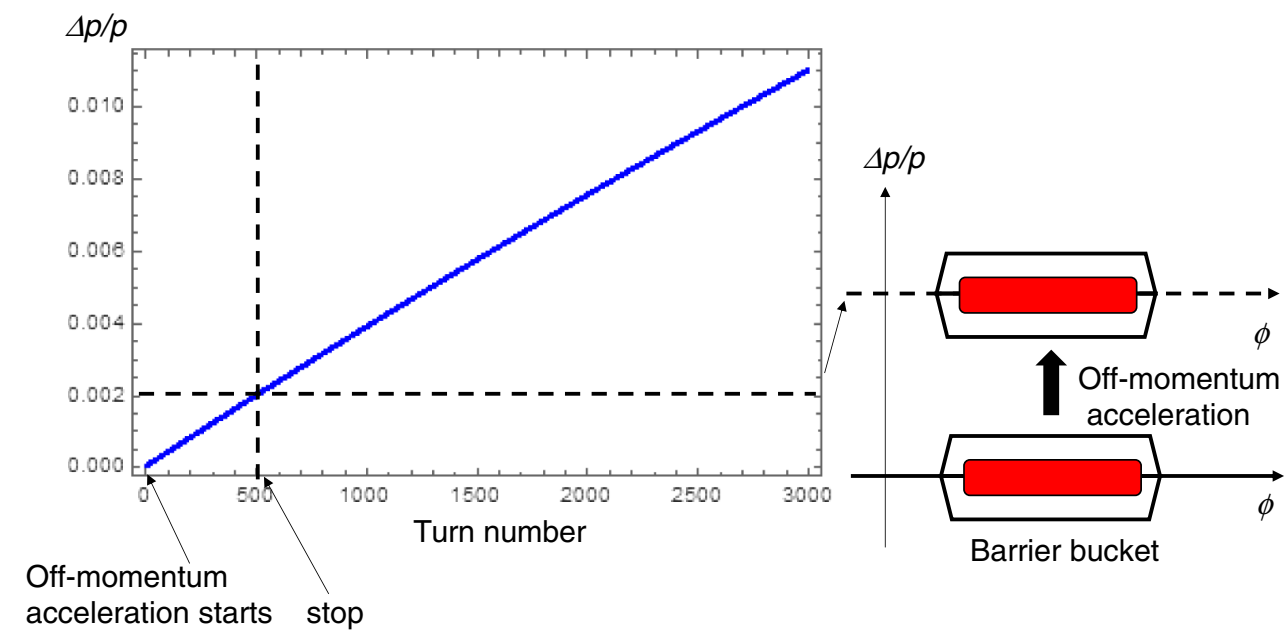

FIG. 4. Evolution of momentum deviation associated with off-momentum deceleration.

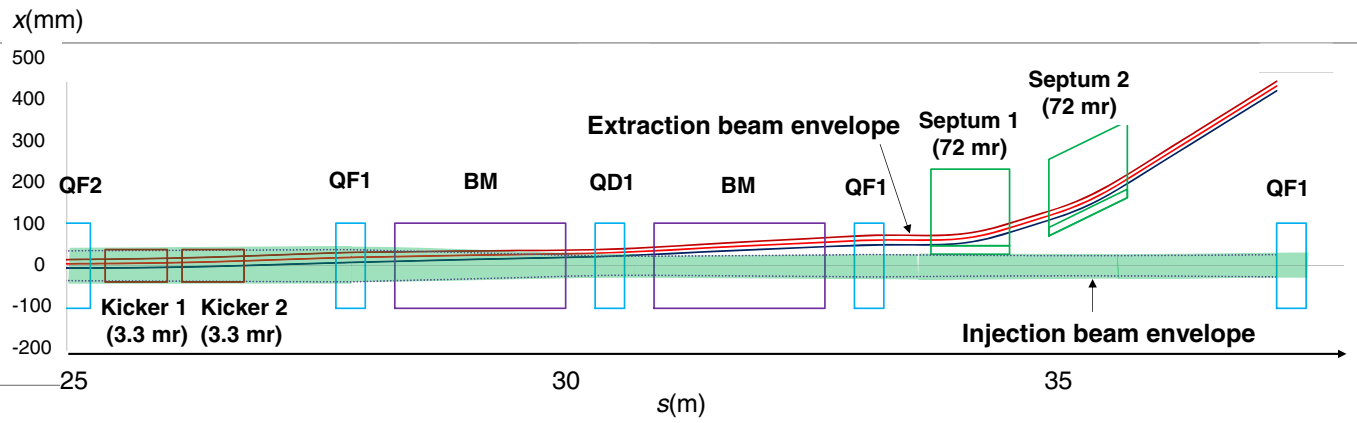

FIG. 5. Beam envelopes in the one-turn extraction region. 


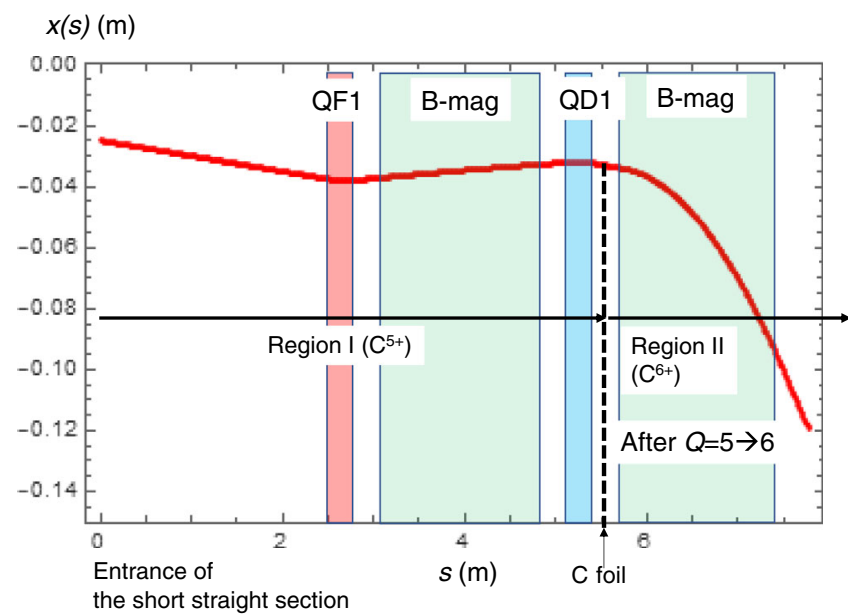

FIG. 6. Extraction orbits of a carbon ion with the momentum deviation of $\left(\Delta p / p_{0}\right)_{I}=-1 \%$ in region I; the last stage orbit in QF1 assumes a nominal gradient.

magnet power supplies to allow one-turn extraction at arbitrary timing are described in Sec. IV.

\section{EXTRACTION ORBIT FOR ENERGY SWEEPING EXTRACTION}

A schematic of the extraction region is shown in Fig. 6. The extraction system simply consists of the stripping foil located between quadrupole magnet (QD1) and bending magnet (BM) and the septum magnets in the 4-m-long straight section. The beam is coasting over the energy sweeping time period, as discussed in Ref. [1]. The beam dynamics of an individual $\mathrm{C}^{5+}$ ion is characterized by its horizontal emittance $\varepsilon_{x}$ and momentum deviation $\Delta p / p$. First, let us consider an orbit of the zero-emittance $\mathrm{C}^{5+}$ ion. After slipping off the barrier bucket, the negative momentum deviation increases rapidly with magnet ramping. The $\mathrm{C}^{5+}$ ion immediately changes to $\mathrm{C}^{6+}$ when penetrating the foil. The equilibrium orbit, $D\left(s_{C}\right) \Delta p / p$, where $s_{C}$ is the location of stripper carbon foil along the beam orbit circumference, arrives at the foil edge fixed at $x=-3 \mathrm{~cm}$, which is cleared by the injected $\mathrm{C}^{5+}$ beam. Details, including the conversion ratio and heat deposition in the foil, are discussed in Sec. IV.

The extraction orbit of the converted $\mathrm{C}^{6+}$ ion is uniquely determined by the effect of the decreasing bending radius through the bending magnet downstream. From the concept of beam rigidity, we know that the converted $\mathrm{C}^{6+}$ ion behaves like a $\mathrm{C}^{5+}$ ion with momentum abruptly reduced by $\Delta p^{\text {effect }} / p_{0}=(5 / 6)-1$ in any magnetic field. Thus, the additional kick at the exit of the bending magnet is written as an integrated vector:

$$
\frac{\Delta p}{p_{0}} \cdot\left(\begin{array}{c}
\rho_{0}\left[1-\cos \left(\ell_{B} / \rho_{0}\right)\right] \\
\sin \left(\ell_{B} / \rho_{0}\right)
\end{array}\right),
$$

where $\rho_{0}$ is the bending radius and $\ell_{B}$ is the magnet length. In this context, the orbit coordinate $s$ beyond the carbon foil is still chosen to be same as that of $\mathrm{C}^{5+}$. A momentum change after charge stripping is ignored, because the momentum loss due to a stripped electron and associated with straggling through the stripping foil is negligible, as shown in Sec. IV. We obtain the momentum deviation $\left(\Delta p / p_{0}\right)_{\mathrm{II}}=\left(\Delta p / p_{0}\right)_{I}+\Delta p^{\text {effect }} / p_{0}$, in region II beyond the stripper foil, where $\left(\Delta p / p_{0}\right)_{I}$ is the momentum deviation in region I. The most striking effect of charge stripping in extraction is the large orbit separation just at the exit of the bending magnet (Fig. 6). This substantially relaxes the septum magnet parameters, such as field strength or physical length. The large orbit separation and negative gradient may pose a specific aperture restriction to the quadrupole magnet (QF1) just after the bending magnet, as discussed in the paragraph below.

In Fig. 6, a typical orbit through regions I and II is shown in red. At first glance, we expect a large orbit deviation from the circulating orbit that is $x=0 \mathrm{~m}$ beyond the exit of the bending magnet, which seems to require a wideaperture ceramic chamber and a specific shape of quadrupole focusing magnet $(\mathrm{QF} 1)$ that can accommodate the extracted beam in the outer region of the magnetic poles. The $\mathrm{C}^{5+} / \mathrm{C}^{6+}$ beam distributions with a finite emittance from the 2.5-m-long straight section to the 4-m-long straight section are shown in Fig. 7. The extraction center

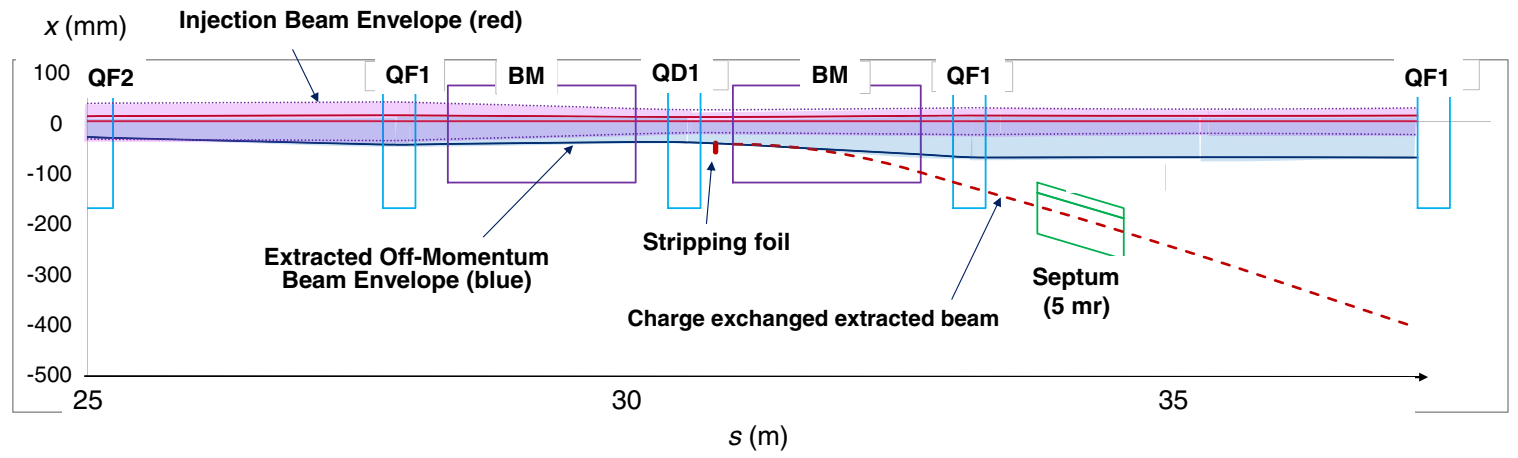

FIG. 7. Beam envelope and beam centroid in energy sweep extraction. 
orbit of the $\mathrm{C}^{6+}$ beam is shown in the same figure. It is determined by the magnetic fields of the downstream QF1 magnet and septum magnet, as discussed more in Sec. IV D. The septum magnet occupies the long straight section, which is ramped in a ramping pattern in time similar to the main bending magnet, as discussed in Sec. IV.
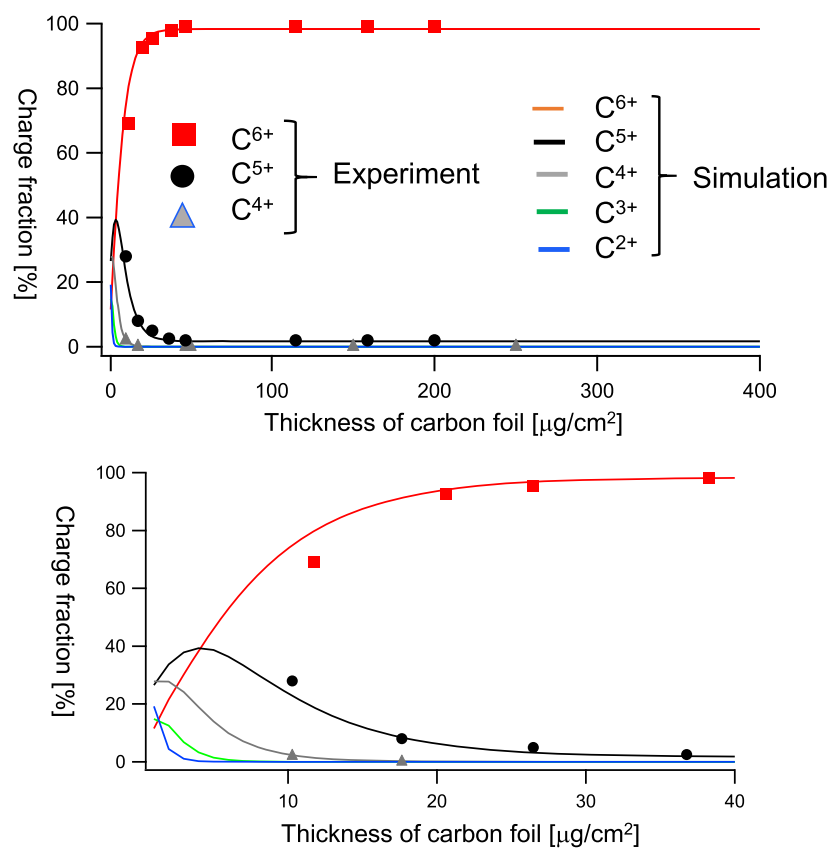

(a)

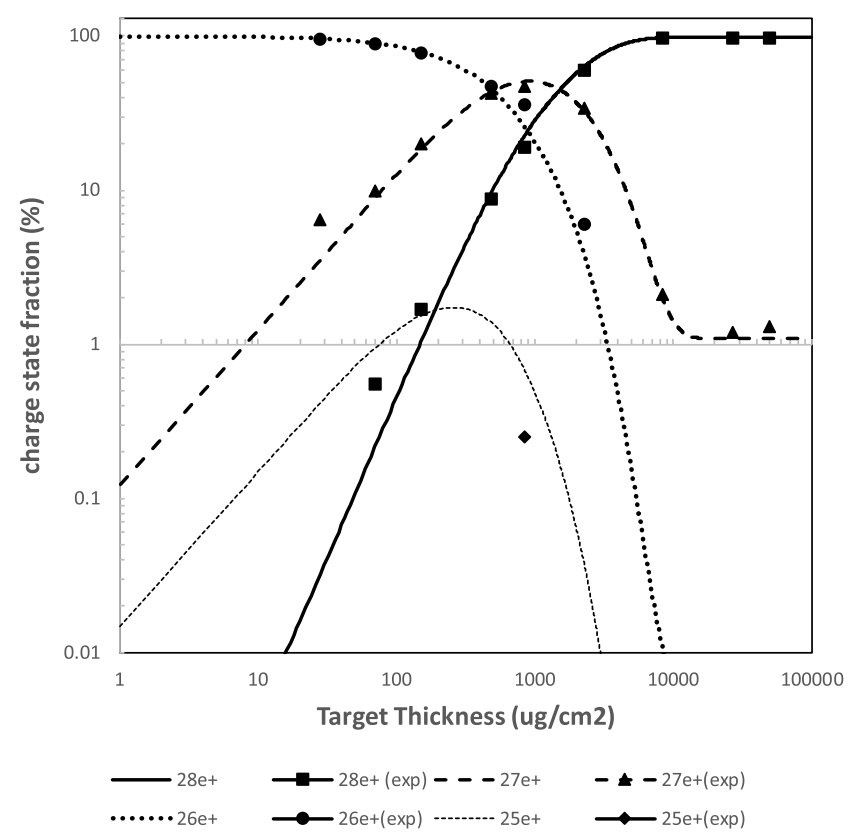

(b)

FIG. 8. (a) Charge conversion efficiency of LISE++ simulation and existing experimental results. The lower panel is an enlargement in the thin foil region. Plotting of the simulation results starts from $1 \mu \mathrm{g} / \mathrm{cm}^{2}$. (b) The measured charge state distribution vs carbon-foil target thickness and simulation results.

\section{INSTRUMENTS}

\section{A. Carbon stripper}

Carbon stripper foil has long been used for $\mathrm{H}^{-}$injection into synchrotrons and charge exchange extraction from proton cyclotrons. Currently, carbon foil is commonly used to generate $\mathrm{C}^{6+}$ from $\mathrm{C}^{4+}$ after the low-energy injector linac (6-8 MeV/au) and before the driver synchrotron in a carbon therapy. Charge conversion efficiency of almost $100 \%$ has been reported. Carbon stripper foil should be feasible for charge exchange at the extraction energy in ESCORT. To confirm the feasibility of the foil, computer simulation using ETACH4 in LISE++ code [7] was carried out.

\section{Charge conversion}

The reliability of the code was checked by comparing calculation results with experimental results [8] before starting extensive simulations. Good consistency between simulation results using ETACH4 in LISE++ and experimental results was confirmed. Figure 8(a) shows the charge conversion efficiency as a function of carbon thickness $\left(\mu \mathrm{g} / \mathrm{cm}^{2}\right)$ for a $\mathrm{C}^{2+}$ ion of $5.924 \mathrm{MeV} / \mathrm{au}$, and the LISE++ result agrees well with the experimental results. This means the justification of the simulation code in a low-energy region. To confirm its availability in a higher-energy region, the experimental results for $\mathrm{Ni}^{26+}(74 \mathrm{MeV} / \mathrm{au})$ in carbon foils [9] are compared with the simulation results in Fig. 8(b). One finds fairly good agreements. We may conclude that ETACH4 in LISE++ is available in our energy region of present interest.

Next, ETACH in LISE++ was used to calculate the charge conversion ratio from $\mathrm{C}^{5+}$ to $\mathrm{C}^{6+}$ as a function of carbon foil thickness for different energies (Fig. 9). Above a foil thickness of $1000 \mu \mathrm{g} / \mathrm{cm}^{2}$, the conversion efficiency was saturated at close to $100 \%$ for the energy range of interest. The energy sweeping extraction is expected to work with stripper carbon foil of $1000 \mu \mathrm{g} / \mathrm{cm}^{2}$ thickness being set at the expected position.

\section{Energy loss in the stripper foil}

The energy loss of a $\mathrm{C}^{5+}$ ion associated with penetration through the foil is a major concern and is calculated by using SRIM or ETACH4 in LISE++ code. The SRIM calculation shows that $(d E / d x)_{\text {elect }} \sim(1.3-1.1) \times$ $10^{-1} \mathrm{MeV} /\left(\mathrm{mg} / \mathrm{cm}^{2}\right)$ for a carbon ion of 200 $300 \mathrm{MeV} / \mathrm{au}$. Assuming carbon foil $1000 \mu \mathrm{g} / \mathrm{cm}^{2}$ thick, the energy loss of a carbon ion associated with penetration is $\Delta E=1000\left[\mu \mathrm{g} / \mathrm{cm}^{2}\right] \times 1.2 \times 10^{5} \times 10^{-3}[\mathrm{eV}] /$ $\left[\mathrm{mg} / \mathrm{cm}^{2}\right]=120[\mathrm{keV}]$. From the total number of carbon ions per shot of $N \sim 10^{8}$ and a repetition rate of $f=10$, the power deposited on the foil is $P=\Delta E N f=1.2 \times$ $10^{5} \times 1.6 \times 10^{-19} \times 10^{8} \times 10[\mathrm{~J} / \mathrm{s}] \sim 20[\mu \mathrm{W}]$. The total magnitude of the power deposited on the foil is small, suggesting that forced cooling is unnecessary. 

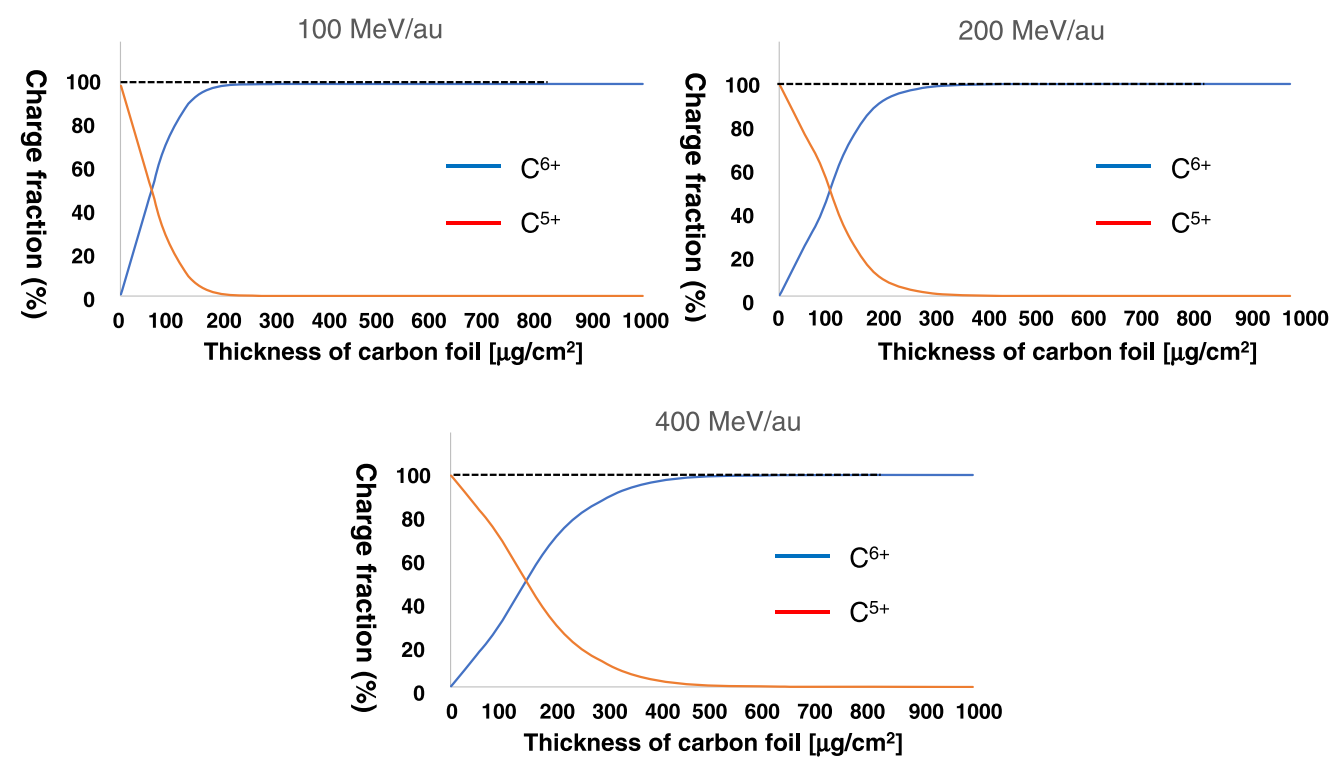

FIG. 9. Charge conversion efficiency for $\mathrm{C}^{5+}$ of 100,200 , and $400 \mathrm{MeV} / \mathrm{au}$.

\section{Possible layout of the stripper foil configuration}

Figure 10 shows a schematic of the vacuum chamber in which the stripper foil mounted on the L-shape frame is embedded together with the typical orbits of a circulating $\mathrm{C}^{5+}$ ion, an off-momentum $\mathrm{C}^{5+}$ ion, and a converted $\mathrm{C}^{6+}$ ion. The carbon foil may be a consumable due to structure damages and be replaced at fixed intervals.

\section{B. Kicker magnet and its power supply}

As mentioned in Secs. II and III, a kicker magnet is not needed for the extraction scheme described in Sec. III (energy sweep extraction) but needed for the scheme described in Sec. II (energy-varying one-turn extraction).

Kicker magnets themselves are nothing new, and any explanation for this will be unnecessary. However, the power supply for the present kicker is specific. An equivalent circuit of the kicker power supply to meet the demand is shown in the upper part of Fig. 11. Initially, the switch (SW-1) is turned on at $\mathrm{S} 1_{\text {on }}$ (see the lower figure), and the high-voltage power

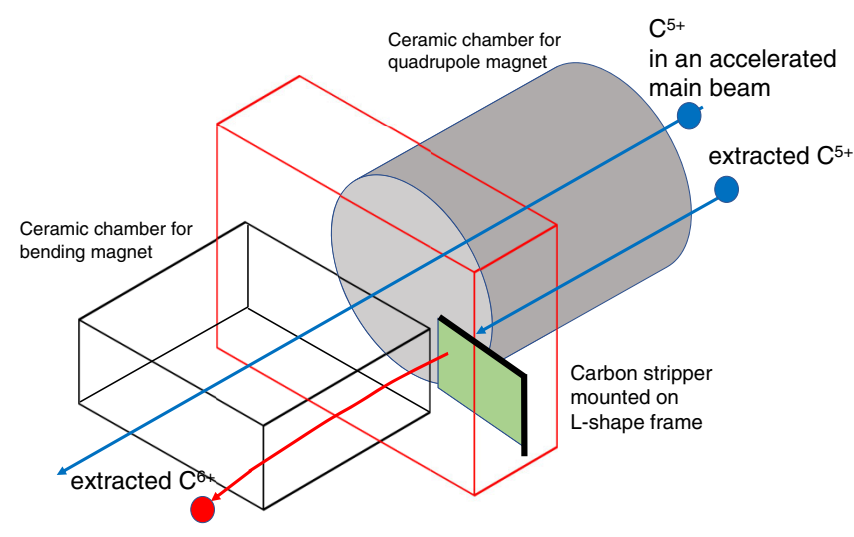

FIG. 10. Stripper foil configuration. supply (HV) begins to charge the pulse forming network (PFN) consisting of two parallel $25 \Omega$ coaxial cables $20 \mathrm{~m}$ long as shown in the lower figure in Fig. 11. When the voltage of the PFN reaches the value corresponding to the desired extraction energy, SW-1 is turned off at $\mathrm{S}_{\text {off }}(1)$. Then, switch SW-2 is turned on at the extraction timing ( $\left.\mathrm{S} 2_{\text {on }}\right)$ determined from irradiation planning. The circuit current flows to the kicker magnet through the two parallel $25 \Omega$ coaxial cables and reflects at the short end. The reverse current with the opposite voltage goes though the kicker magnet, transmission cable, SW-2, PFN, diode (D), and generates losses at the $12.5 \Omega$ matching resistor (R). In the next acceleration period, the PFN is charged again at $S 1_{\text {on }}$ and stops when the PFN voltage reaches the required value at $\mathrm{S}_{\text {off }}(2)$. This charging PFN voltage can be changed pulse to pulse so as to match the varying extraction energy. Therefore, changing the turn-off timing of SW-1 to match the extraction energy is mostly crucial for this circuit.

It is noted that the kicker will be also used as an abort kicker when a fraction of particles remains in the driver ring just after energy sweep extraction. A beam dump placed on the high-energy beam transport line (not shown) equipped with a beam switcher magnet accepts the extracted beam.

\section{Septum magnet and power supply}

A septum magnet is needed not only for energy-varying one-turn extraction but also for energy sweep extraction. The magnets themselves are the same for both extractions, but their power supplies are different.

The septum magnet for energy-varying one-turn extraction must be excited with the high current, resulting in a large kick angle. As the extraction energy is changed pulse to pulse, the peak current through the septum coil should be changed so as to match the extraction energy. 

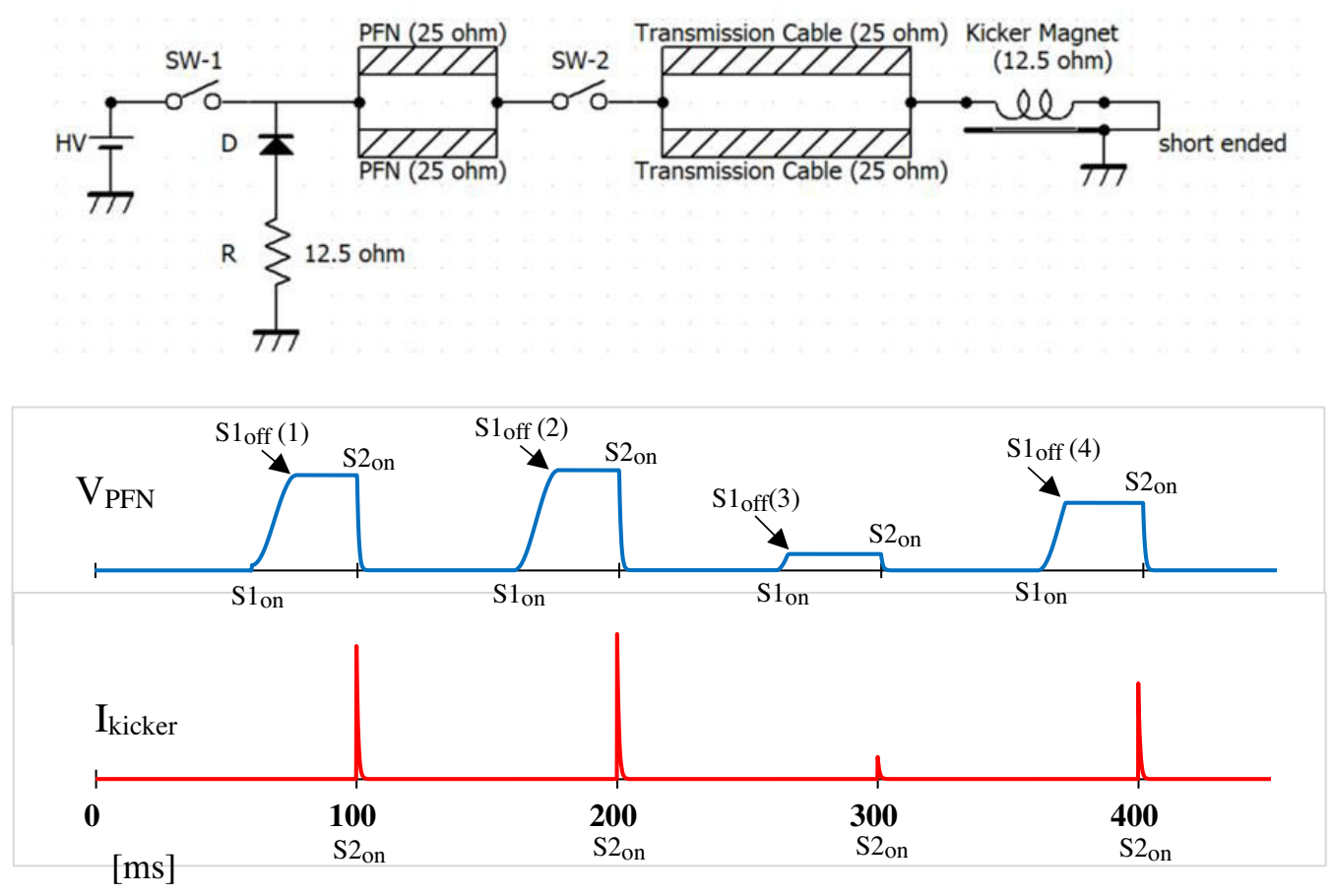

FIG. 11. Upper: equivalent circuit of the kicker magnet power supply; lower: charged voltage of the PFN (25 $\Omega)$ and excitation current of the kicker magnet.
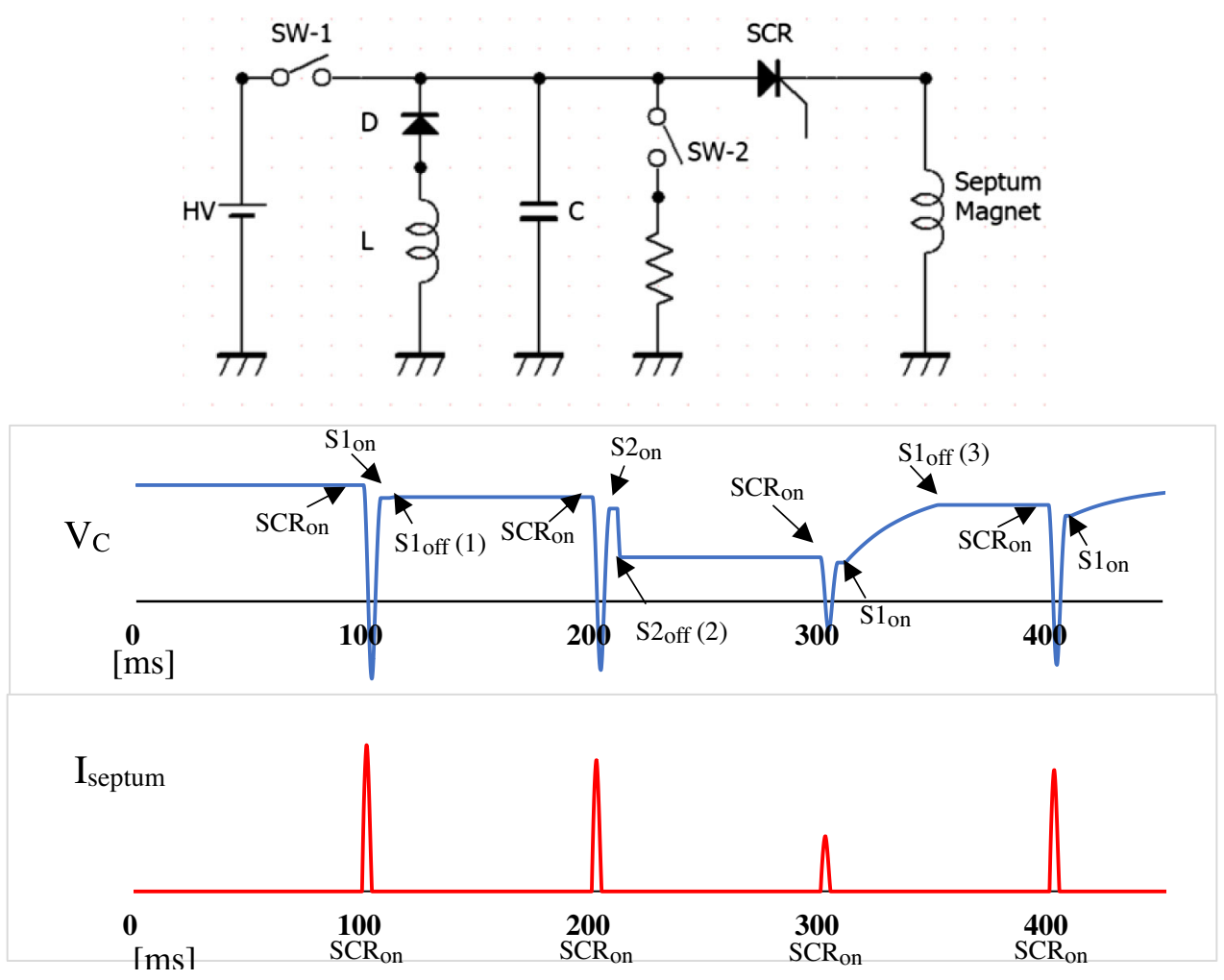

FIG. 12. Upper: circuit architecture of the septum magnet; lower: charging voltage of capacitor (C) and excitation current of septum magnet for energy-varying one-turn extraction. 
The equivalent circuit of the power supply for the septum magnet for energy-varying one-turn extraction is shown in the upper figure in Fig. 12. To make the charging and discharging procedure clear, let us see how it works in a sequential operation of 4. First, as shown in the lower figure in Fig. 12, the capacitor (C) with capacitance $C$ is recharged from the power supply (HV) by turning on the switch (SW-1) at the timing of $S 1_{\text {on }}$. When the capacitor voltage achieves the expected value, $\mathrm{SW}-1$ is turned off at $S 1_{\text {off }}(3)$. At the expected timing $\left(\mathrm{SCR}_{\mathrm{on}}\right)$, the thyristor (SCR) is turned on, and the current flows from the capacitor to the septum magnet with inductance $L$. When the current direction reverses, the thyristor (SCR) is automatically turned off, and the reverse current flows through the diode (D) and inductor (L) until the capacitor (C) is recharged. If the charged voltage of the capacitor is less than a requested value in the next cycle, the capacitor begins to be charged by turning on SW-1 at the timing of $\mathrm{S} 1_{\text {on }}$ as seen in the middle of Fig. 12 (first, third, and fourth cycle). On the contrary, the charged voltage of the capacitor exceeds the requested value in the time period of the second cycle, and there the charge of the capacitor is discharged by turning on $\mathrm{SW}-2$ at $\mathrm{S} 2_{\text {on }}$, which is turned off when the capacitor voltage achieves the expected value at $S 2_{\text {off }}(2)$. Then, the thyristor (SCR) is turned on at the next timing $\left(\mathrm{SCR}_{\mathrm{on}}\right)$. Therefore, changing the turn-off timing of SW-1 or SW-2 following the extraction energy is mostly crucial for this circuit, too. As the pulse profile of the septum current is half sinelike with a width of a few milliseconds, Joule heating generated in the septum conductor is acceptable.

There are two circuit architectures to drive the septum for energy sweeping extraction, that allow one to follow the ramping pattern of the fast-cycling main bending magnet: (i) a half-sine current driving circuit with the dc bias current circuit and (ii) dual polarity current driving circuit with the dc bias current circuit.

In (i), it is assumed that the energy sweep extraction starts from the middle of the acceleration cycle, which is


FIG. 13. Upper: circuit architecture for the septum magnet; lower: LT spice simulation results, charging voltage of capacitor (C1), recharging current and excitation current of septum magnet for energy sweeping extraction, where [Sn] indicates the trigger timing of SCR “Sn," where Lm of $24 \mu \mathrm{H}$, Rm of $0.38 \mathrm{~m} \Omega$, and $\mathrm{C} 1$ of $15.92 \mathrm{mF}$ are assumed. 
specified by " $t_{c}$." The septum magnet must be always excited in the same ramping pattern as that of the main bending magnet from $t_{c}$, since the beam orbit downstream has to be placed on the designed center orbit over any possible extraction timing. An equivalent circuit of the power supply is shown in the upper part of Fig. 13. It is noted that the product of capacitance $\mathrm{C} 1$ and $(\mathrm{La}+\mathrm{Lm})$, where $\mathrm{La}$ and $\mathrm{Lm}$ are the inductance of the choke and septum magnet, respectively, should be the same as the product of $\mathrm{C}$ and $\mathrm{L}$ of the main bending magnet driving circuit, so as to secure the same field ramping pattern. As $\mathrm{Lm}$ is very small in most cases, a choke with large $\mathrm{La}$ is connected to the septum magnet coil in series so as to satisfy the condition $\mathrm{C} 1(\mathrm{La}+\mathrm{Lm})=\mathrm{LC}$. The dc bias current circuit seen in Fig. 13 to supply the necessary current at " $t_{c}$ " is indispensable. As shown in the lower part of Fig. 13, where the pulse profiles are LT Spice simulation results, the thyristor switch $\mathrm{S} 3$ is turned on at " $t_{c}$." The current from $\mathrm{C} 1$ flows to the septum magnet coil and stops when the voltage of $\mathrm{C} 1$ reaches the negative peak voltage. Then, the thyristor switch S2 is turned on, and the current from $\mathrm{C} 1$ flows though $\mathrm{Lr}$ until $\mathrm{C} 1$ is charged to the positive peak voltage. As this voltage is slightly lower than the initial charged voltage due to the circuit loss, $\mathrm{S} 1$ is turned on until the voltage reaches the required initial value.

In (ii), energy sweep extraction over the entire acceleration period is assumed, where the current profile of the septum magnet completely follows that of the bending magnet through the acceleration period. This is simply achieved by connecting the symmetric pulse circuit with the negatively charged capacitor $\mathrm{C} 1$ to the circuit for (i), and both SCR groups (S1-S3) are turned on alternately every $50 \mathrm{~ms}$, though its details are not described here.

An operational time period of this septum magnet is much larger than that of the septum for energy-varying one-turn extraction. However, the bending angle required for the energy sweeping extraction septum is rather small (about $6 \mathrm{mrad}$ ) with the help of charge stripping and enhanced bending in the bending magnet downstream; Joule heating generated in the septum conductor is still estimated to be acceptable. The architecture (i) may be likely in practical uses, because a too large tumor will be not assumed.

\section{Quadrupole magnet (QF1)}

For the energy sweeping extraction, we focus on the cross section of the focusing quadrupole (QF1) located just after the bending magnet, because the extracted beam propagates with a large orbit separation from the magnet center of $11-13 \mathrm{~cm}$. This size demands a large-aperture magnet. Usually, such a large-aperture quadrupole magnet is undesirable owing to its cost. Instead, a quadrupole magnet as shown above has been considered. This magnet is a normal quadrupole magnet with a slightly longer magnetic flux path, where some space between the poles

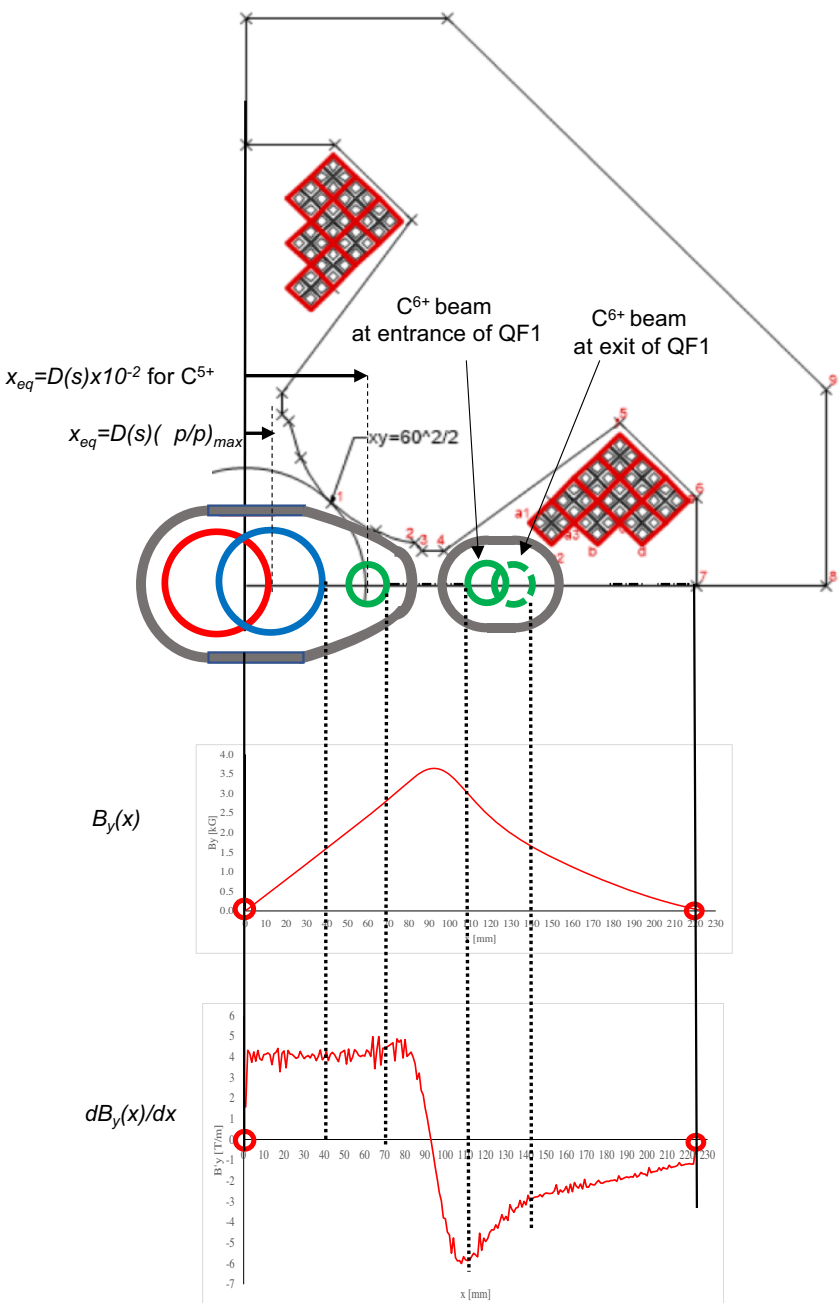

FIG. 14. Specific focusing quadrupole magnet QF1 with ceramic chambers (gray) and field distributions. Blue and red circles mean outer edges of the injected beam with a momentum spread of $|\Delta p / p|=0.1 \%$.

and excitation coil is reserved to accommodate the ceramic chamber for the extracted $\mathrm{C}^{6+}$ beam. Its overall size is large to meet the requirements for extraction, as seen in Fig. 14. The magnetic fields on the beam orbit give Lorentz force to push back the beam centroid toward the magnet center and nonlinear defocusing forces in the horizontal direction on particles around the centroid. Although the $\mathrm{C}^{6+}$ beam is kicked outward by about $10 \mathrm{mrad}$, its extraction is easily realized by the single septum magnet to give a 5 mrad kick angle, as shown in Fig. 7. In addition, the nonlinear defocusing effects are rather small.

\section{SUMMARY}

Two methods of beam extraction from the fast-cycling induction synchrotron (ESCORT driver ring) were proposed with the maximum use of the localized large flat dispersion function in the extraction region and barrier 
bucket induction acceleration in the ESCORT driver ring. Details of one-turn energy-varying beam extraction assisted by the off-momentum orbit, which performs a role similar to a bump orbit, were discussed. We emphasize that an irradiation $\mathrm{C}^{6+}$ bunch can be extracted at a desired timing, substantially reducing the role of kickers.

The feasibility of the energy sweeping extraction using stripper carbon foil, which is a simple low-cost device, was examined. The $\mathrm{C}^{6+}$ ions, which were converted from $\mathrm{C}^{5+}$ ions, were bent greatly by the bending magnet downstream and propagated through the following focusing magnet.

Because the entire magnet system of ESCORT, including that of kickers and septum magnets, is operated in the fastcycling synchrotron mode, the beam orbit is always common for any energy. Typical circuit examples for the kicker and septum to allow this have been described. Thus, the irradiation beam delivered with required energy at $10 \mathrm{~Hz}$ can be guided to a patient or target, assuming that the high-energy beam transport, except for the correction magnet system for real-time target tracking, is operated in the same ramping mode.

Last, it is noted that barrier bucket beam handling for energy sweep extraction in the 70-m-long ESCORT ring taken here will be managed with more practical parameters than that in the original proposal [1] without any change of its essential features. For instance, induction barrier voltage pulses with a rising or falling time of $50 \mathrm{~ns}$ instead of $35 \mathrm{~ns}$ are assumed. Details will be described in a forthcoming paper [3], where an energy sweeping compact carbon therapy is reviewed including the long-life laser ablation ion source, gantry-free fast-feedback irradiation system, real-time dose profile monitoring system, and real-time tumor profile monitoring system.

\section{ACKNOWLEDGMENTS}

The lattice function calculation was carried out by Leo Kwee Wah (Malaysian Nuclear Agency).
[1] L. K. Wah, T. Monma, T. Adachi, T. Kawakubo, T. Dixit, and K. Takayama, Compact hadron driver for cancer therapies using continuous energy sweep scanning, Phys. Rev. Accel. Beams 19, 042802 (2016).

[2] K. Takayama, T. Yoshimoto, M. Barata, L. K. Wah, L. Xingguang, T. Iwashita, S. Harada, T. Adachi, T. Arai, D. Arakawa, H. Asao, E. Kadokura, T. Kawakubo, H. Nakanishi, Y. Okada, K. Okamura, K. Okazaki, A. Takagi, S. Takano, and M. Wake, Induction acceleration of heavy ions in the KEK digital accelerator: Demonstration of a fast-cycling induction synchrotron, Phys. Rev. Accel. Beams 17, 010101 (2014).

[3] K. Takayama, T. Adachi, T. Kawakubo, K. Okamura, N. Munemoto, S. Takano, E. Kadokura, J. Hasegawa, K. Horioka, Leo K. Wah, T. Dixit, and A. Deshpande, The energy sweeping compact hadron therapy (ESCORT) integrated with real time monitoring of the tumor image/dose profile and fast-feedback irradiation (to be published).

[4] K. Takayama, K. Okamura, and T. Kawakubo, Patent No. 01520, 2020.

[5] M. Umezawa, F. Ebina, Y. Fujii, K. Matsuda, K. Hiramoto, K. Umegaki, and H. Shirato, Development of compact proton beam therapy system for moving organs, Hitachi Review 64, 86 (2015).

[6] A. Koide, J. Kataoka, T. Masuda, S. Mochizuki, T. Taya, K. Sueoka, L. Tagawa, K. Fujieda, T. Maruhashi, T. Kurihara, and T. Inaniwa, Precision imaging of $4.4 \mathrm{MeV}$ gamma rays using 3-D position sensitive Compton camera, Sci. Rep. 8, 8116 (2018).

[7] O. B. Tarasov and D. Banzin, LISE++: Radioactive beam production with in-flight separators, Nucl. Instrum. Methods Phys. Res., Sect. B 266, 4657 (2008).

[8] Y. Sato, A. Kitagawa, M. Muramatsu, T. Murakami, S. Yamada, C. Kobayashi, Y. Kageyama, T. Miyoshi, H. Ogawa, H. Nakabushi, T. Fujimoto, T. Miyata, and Y. Sano, Charge fraction of $6.0 \mathrm{MeV} / \mathrm{au}$ heavy ions with a carbon foil: Dependence on the foil thickness and projectile atomic number, Nucl. Instrum. Methods Phys. Res., Sect. B 201, 571 (2003).

[9] H. Rothard, J.-P. Grandin, M. Jung, A. Clouvas, J.-P. Rozet, and R. Wunsch, Pre-equilibrium charge state distributions of $\mathrm{Ni}^{26+}(74 \mathrm{MeV} / \mathrm{u})$ in carbon foils, Nucl. Instrum. Methods Phys. Res., Sect. B 132, 359 (1997). 